\title{
Oxytocin as a pharmacological target for benign prostatic hyperplasia
}

\author{
Betty Exintaris ${ }^{1}$, Sophie Lee ${ }^{2}$, Johanna Hammar ${ }^{2}$, Jenna Kraska², Melissa Papargiris², \\ Michael Whittaker ${ }^{3}$, Gail Risbridger ${ }^{2}$, Stuart Ellem ${ }^{2}$, Ralf Middendorff ${ }^{4}$
}

\begin{abstract}
${ }^{1}$ Drug Discovery Biology, Monash Institute of Pharmaceutical Sciences, Australia, ${ }^{2}$ Department of Anatomy and Developmental Biology, Monash Biomedicine Discovery Institute, Monash University, Australia, ${ }^{3}$ ARC Centre of Excellence in Convergent Bio-Nano Science \& Technology, Monash Institute of Pharmaceutical Sciences, Australia, ${ }_{4}^{4}$ Institute of Anatomy and Cell Biology, Justus Liebig University, Giessen, Germany
\end{abstract}

\section{Background}

The pathogenesis of Benign Prostatic Hyperplasia (BPH) is associated with both the non-malignant growth of the prostate (static component), and increased smooth muscle tone (dynamic component) which can lead to irritative and obstructive lower urinary tract symptoms, affecting the quality of life of patients. A recent study has shown that the levels of circulating oxytocin is significantly upregulated in men with (BPH), and exogenous oxytocin significantly increased the proliferation of prostatic fibroblasts [1]. In this study, we investigated whether oxytocin regulates the smooth muscle tone of the human prostate gland.

Methods

Samples of human prostate were collected from the transition (TZ) and peripheral zone (PZ) of men undergoing radical prostatectomy. Immunohistochemistry was used to confirm the presence and localization of the oxytocin receptor (OXTR). Tension recordings were obtained using organ bath techniques, with specimens incubated with increasing concentrations of oxytocin $(0.1 \mathrm{nM}$ to $10 \mu \mathrm{M})$. The basal tension $(\mathrm{mN})$, amplitude $(\mathrm{N} / \mathrm{g})$ and frequency of spontaneous contractions were quantified.

Results

Using Immunohistochemistry, OXTR was found to be widely expressed in both the epithelial and stromal compartment of $\mathrm{TZ}$ and PZ specimens of human prostate $(\mathrm{n}=10)$. Application of exogenous oxytocin to $\mathrm{TZ}$ specimens significantly augmented the frequency of spontaneous contractions at $10 \mu \mathrm{M}$ by $70 \pm 21 \%$ when normalized to baseline frequency ( $\mathrm{p}$ $<0.05, \mathrm{n}=8$ ). The half-maximal increase in frequency (EC50) occurred at $300 \mathrm{nM}$. A similar efficacy was observed in patient matched $\mathrm{PZ}$ specimens $(\mathrm{n}=4)$.

Conclusion

We have established that oxytocin is able to induce contractility of the human prostate in both the $\mathrm{TZ}$ and $\mathrm{PZ}$ of the human prostate. Given that patients with BPH have elevated levels of oxytocin and that oxytocin has previously been shown to induce proliferation of prostate fibroblasts, is widely expressed in both the prostate stroma and epithelium, and is able to induce muscle contractility, targeting of the OXTR and associated downstream signalling is an attractive prospect in designing new and novel BPH pharmacotherapies.

1. Xu, H., et al., Oxytocin: its role in benign prostatic hyperplasia via the ERK pathway. Clin Sci (Lond), 2017. 131(7): p. 595-607. 